\title{
Aprendizaje indagativo sobre los cambios físicos y químicos en la formación inicial del profesorado de secundaria
}

\author{
Iñigo Rodríguez-Arteche ${ }^{1,2}$, Ana I. Bárcena2 ${ }^{2}$ David Rosa² \\ y M. Mercedes Martínez-Aznar ${ }^{2}$ \\ ${ }^{1}$ Facultad de Ciencias Jurídicas y Sociales. Univ. Rey Juan Carlos. ${ }^{2}$ Facultad de Educación - \\ Centro de Formación del Profesorado. Universidad Complutense de Madrid. España
}

[Recibido el 5 de noviembre de 2018, aceptado el 10 de abril de 2019]

La indagación conforma un enfoque beneficioso para el aprendizaje de las ciencias, pero demanda un diseño cuidadoso de las actividades y del rol del profesor para conjugar un aprendizaje conceptual, procedimental y actitudinal. El artículo se vincula a una propuesta para la asignatura de Didáctica de la Química del Máster en Formación del Profesorado, para formar al futuro profesorado sobre estas metodologías. Así, tras ofrecer vivencias personales sobre el aprendizaje por indagación, el trabajo busca examinar los logros de los estudiantes en la resolución de problemas abiertos sobre el cambio químico. A partir del análisis de los informes escritos individuales, se comprueba una mejoría en la competencia científica de los participantes, especialmente acusada en las dimensiones más conceptuales, y menor en las asociadas al diseño de estrategias experimentales de resolución.

Palabras clave: formación inicial del profesorado; indagación; Metodología de Resolución de Problemas como Investigación (MRPI); problemas abiertos; cambio químico.

\section{Inquiry-based learning about physical and chemical changes during initial high school teacher training}

Inquiry-Based Science Education (IBSE) is an effective approach for learning science, but it demands a careful design of the activities and the role of the teacher to combine a conceptual, procedural and attitudinal learning. This article is linked to a proposal for the Chemistry Education subject of the Spanish Master's in Secondary Education, which aims to train future high school teachers on these methodologies. The subject provides some first-hand opportunities to learn school chemistry by using IBSE. Therefore, this paper seeks to analyze the level of achievement by future teachers in solving open-ended problems about chemical change. The analysis of the individual written reports shows an improvement in the future teachers' scientific competencies, which is especially relevant in the more conceptual dimensions, and less accused in those associated with the design of experimental solving strategies.

Keywords: initial teacher training; inquiry; Methodology of Problem Solving as an Investigation (MPSI); open-ended problems; chemical change.

Para citar el artículo. Rodríguez-Arteche, I., Bárcena, A.I., Rosa, D. y Martínez-Aznar, M.M. (2019). Aprendizaje indagativo sobre los cambios físicos y químicos en la formación inicial del profesorado de secundaria. Ápice. Revista de Educación Científica, 3(2), 1-20. DOI: https:// doi.org/10.17979/arec.2019.3.2.4657

Contacto.inigo.rodriguez@urjc.es, anabarce@ucm.es, darosa@ucm.es,mtzaznar@ucm.es 


\section{Marco teórico}

\section{La indagación como estrategia metodológica}

En las últimas décadas, las metodologías de carácter indagativo (IBSE, inquiry-based science education) están recibiendo un amplio respaldo de la comunidad dedicada a la investigación en didáctica de las ciencias y de las instituciones educativas comunitarias. Existen distintas acepciones sobre el alcance y las características de la indagación (Aguilera et al., 2018), que llevan a plantearnos si estamos hablando de lo mismo al referirnos a este enfoque de enseñanza-aprendizaje (Romero-Ariza, 2017; Windschitl, Thompson y Braaten, 2008) y a comparar sus logros con los correspondientes a los modelos de instrucción directa (Cobern et al., 2010; Kirschner, Sweller y Clark, 2006). Por ello, comenzamos indicando que en este artículo se asume una visión amplia de la «indagación», que incluye el uso y la construcción de modelos y argumentos como elementos centrales de la práctica científica (Acevedo, García-Carmona, Aragón y Oliva, 2017; Jiménez-Aleixandre y Puig, 2012). De este modo, consideramos la siguiente definición de Crawford (2007):

[La indagación implica] comprender las diversas formas en que los científicos realizan su trabajo, valorar el potencial de las observaciones, habilidad para formular preguntas investigables e hipótesis, utilizar distintos tipos de datos para buscar patrones y confirmar o rechazar las predicciones, construir y defender modelos y argumentos, evaluar explicaciones alternativas y lograr una mejor comprensión del carácter provisional y evolutivo de la ciencia y su origen en la actividad humana, el contexto y la cultura en que se desarrolla y utiliza. (p. 614)

Autores como Prince y Felder (2006) han aportado clasificaciones de las estrategias indagativas según el tipo de tareas propuestas (realización de proyectos, análisis de controversias, resolución de problemas, etc.), variantes que comparten el rasgo del «andamiaje»: la ayuda del profesor a los estudiantes para completar tareas que no podrían resolver por sí mismos (Hmelo-Silver, Duncan y Chinn, 2007). Así, el andamiaje es un proceso dinámico de interacción entre docentes y estudiantes, a través de estrategias como las pistas, la retroalimentación, la modelización, la formulación de preguntas o la instrucción en momentos específicos (Van de Pol, Volman y Beishuizen, 2010).

Dentro del enfoque indagativo, el aprendizaje basado en problemas (PBL, problem-based learning) describe un entorno donde los problemas, retos para los que no hay soluciones evidentes, regulan el aprendizaje (Gil-Pérez y Martínez-Torregrosa, 1983; Hung, 2016). Ello exige un diseño cuidadoso de estas actividades, en términos del ajuste progresivo de su dificultad, del andamiaje o ayuda proporcionada por profesores y compañeros (Domènech, 2013), de los factores emocionales asociados a su resolución (Bevins y Price, 2016) y de la cohesión de los conocimientos teóricos y prácticos en todo el proceso (Crujeiras y Jiménez-Aleixandre, 2015).

El último de los elementos anteriores supone una de las demandas más insistentes por parte de los miembros del área, e implica que la indagación no solo se plantee como «fin» en sí misma (Abd-El-Khalick et al., 2004). Es decir, además de ser útil para el desarrollo de destrezas investigativas y la comprensión de aspectos asociados a la práctica científica, conviene contemplar la indagación como "medio» para el cambio conceptual (Barrow, 2006; Ibáñez y Martínez-Aznar, 2005). Así, una de las estrategias coherentes con una visión actual de la Naturaleza de la Ciencia es la de plantear secuencias de actividades o problemas abiertos (Hernández, Couso y Pintó, 2015; Ibáñez y Martínez-Aznar, 2007) que permitan la evaluación y reconstrucción progresiva de los marcos teóricos y modelos propuestos (Kang, Windschitl, Stroupe y Thompson, 2016). 
Para promover los objetivos anteriores, resulta necesario estructurar los procesos de enseñanza-aprendizaje. Con este fin, existen modelos formativos como las $5 \mathrm{E}$ (Bybee et al., 2006) y otros más específicos del aprendizaje basado en problemas. Entre los últimos, para este trabajo se considera la Metodología de Resolución de Problemas como Investigación -MRPI- (Gil-Pérez y Martínez-Torregrosa, 1983), una estrategia que ha proporcionado buenos resultados en diferentes disciplinas y contextos escolares (Ibáñez y Martínez-Aznar, 2005, 2007; Martínez-Aznar y Bárcena, 2013; Rodríguez-Arteche, Martínez-Aznar y Garitagoitia, 2016).

La MRPI consta de cinco fases vinculadas a Dimensiones de la Competencia científica (DC) y propone un trabajo cíclico y dinámico en grupos cooperativos, perfectamente adaptable a situaciones de lápiz y papel o de naturaleza experimental, y a problemas abiertos de diferente tipo. Estas fases, descritas a continuación y mostradas en la Figura 1, deben entenderse de forma flexible (Rodríguez-Arteche y Martínez-Aznar, 2016a), como un andamiaje de tipo «estructurador» (Reiser, 2004) de los procesos de aprendizaje:

DC1. Análisis cualitativo del problema. Supone trabajar sobre los conceptos y modelos implicados y reformular el problema en términos operativos.

DC2. Emisión de hipótesis, en base al marco teórico.

DC3. Diseño de estrategias de resolución. Abarca la identificación y el control de las variables y la toma de decisiones para resolver los problemas.

DC4. Resolución del problema, donde la verbalización de los procesos y el registro y tratamiento de los datos cobran especial importancia.

DC5. Análisis de resultados, con referencia a las hipótesis, al marco teórico y a nuevos problemas susceptibles de ser investigados.

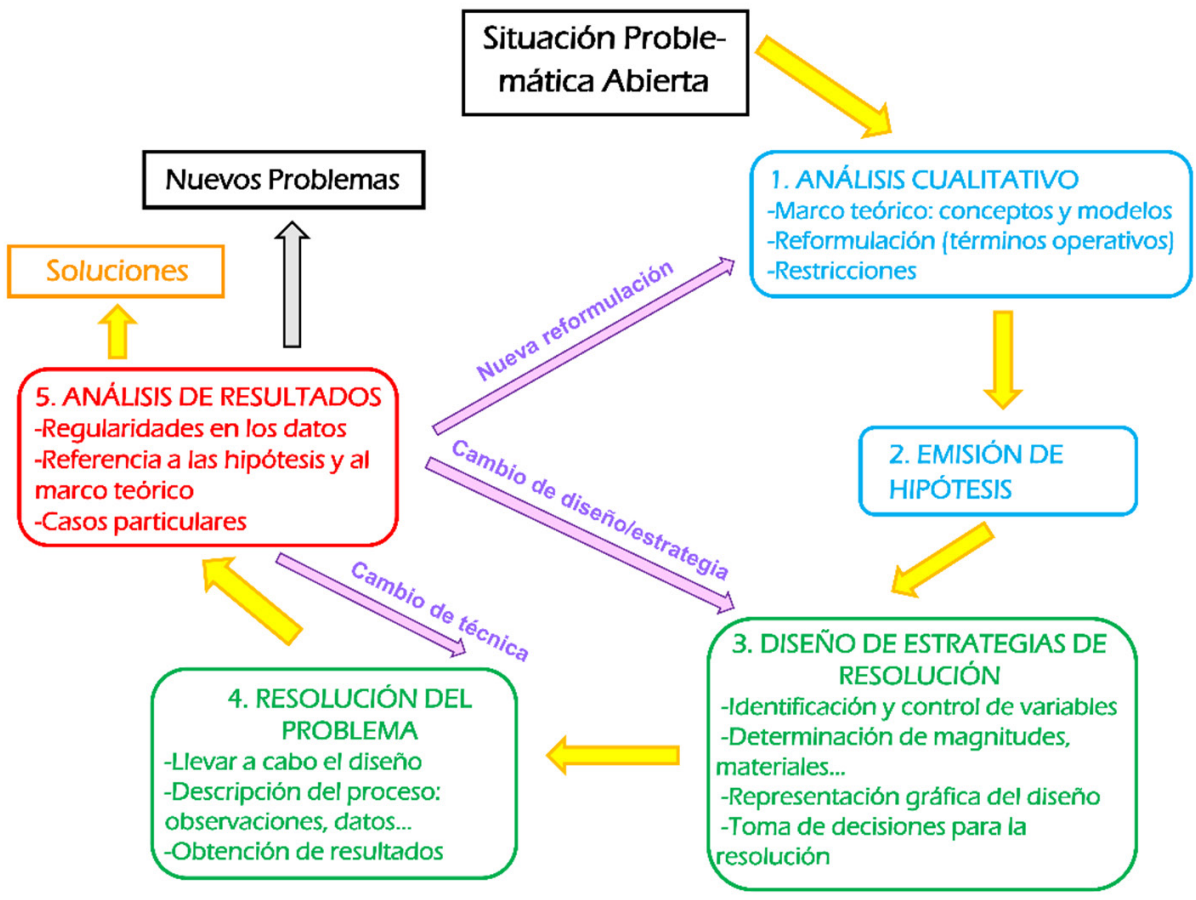

Figura 1. Esquema de la Metodología de Resolución de Problemas como Investigación (MRPI) como proceso cíclico y flexible 


\section{La indagación como problema profesional en la formación inicial del profesorado}

A pesar de los beneficios reconocidos sobre la indagación, su transferencia a las aulas escolares es más bien minoritaria (Capps y Crawford, 2013), hecho que puede relacionarse con las creencias profesionales y con una comprensión superficial de sus características (Wheeler, Bell, Whitworth y Maeng, 2015). En relación con lo primero, los planteamientos indagativos conllevan un cambio en los roles de estudiantes y profesores que, en ocasiones, puede dificultar la supervisión del trabajo del alumnado. Además, este enfoque contrasta con la práctica docente tradicional, y puede generar un cierto rechazo inicial (Rodríguez-Arteche y Martínez-Aznar, 2016b). En relación con lo segundo, se sigue percibiendo una cierta asociación de la indagación con el aprendizaje por «descubrimiento» (Kirschner et al., 2006), así como su identificación con una estrategia muy dirigida a los procedimientos científicos y no tanto al aprendizaje conceptual. Igualmente, el profesorado en ejercicio y en formación tiende a enfatizar el carácter lúdico de las actividades indagativas, lo que sugiere que las conciben más como un recurso para motivar que como un enfoque promotor de los aprendizajes (Gil-Quílez, Martínez-Peña y Cordero, 2017).

Las dificultades descritas justifican la inclusión de las estrategias indagativas como elemento clave de los programas de formación del profesorado. Para ello, existe un amplio consenso sobre la conveniencia de ofrecer «vivencias» de aprendizaje de corte indagativo -para promover una visión genuina sobre los beneficios y los retos de este enfoque(Pilitsis y Duncan, 2012), y sobre el papel fundamental de la reflexión explícita y de la «retroalimentación» por parte de los formadores (Zembal-Saul, Blumenfeld y Krajcik, 2000).

Por estos motivos, muchos programas formativos (p.ej., Crujeiras, 2017; Toma, Greca y Meneses, 2017; Wheeler et al., 2015) están contemplando la selección y el diseño de propuestas indagativas como "problema profesional» en la formación inicial del profesorado (Martínez-Aznar, Rodríguez-Arteche y Gómez-Lesarri, 2017). Así, centrándonos en el contexto español, para la formación inicial de profesores de educación primaria encontramos trabajos sobre temáticas como los circuitos eléctricos (García-Carmona, Criado y Cruz-Guzmán, 2017), la energía (Martínez-Aznar y Varela, 2009), la flotación (Garrido, 2016), el sistema Sol-Tierra (Martínez-Chico, Jiménez-Liso y López-Gay, 2015) o el cambio químico (Garrido, 2016). En el caso de la formación del profesorado de secundaria, el último de los aspectos anteriores está centrando buena parte del interés (Crujeiras y Jiménez-Aleixandre, 2015; Rodríguez-Arteche y Martínez-Aznar, 2016a, 2016c), probablemente por la complejidad que supone el construir un modelo escolar que haga referencia a los tres niveles de representación del cambio químico, y que relacione adecuadamente la diversidad de conceptos implicados (Martín del Pozo, 2001). Precisamente, la temática del cambio químico es la desarrollada en el presente trabajo.

\section{Aprendizaje sobre el cambio químico a través de la indagación}

El aspecto del cambio químico -frente al cambio físico- corresponde a un «concepto estructurante» de la ciencia escolar (Harlen et al., 2015), una idea central cuya construcción favorece una mayor relación entre los conocimientos y, con ello, permite explicar múltiples fenómenos de nuestro entorno (Aragón, Oliva y Navarrete, 2013), contribuyendo a la alfabetización científica del alumnado. Además, la enseñanza-aprendizaje sobre el cambio químico supone una oportunidad para abordar los tres niveles de representación de la química (Johnstone, 1982): el «macroscópico», que comprende el ámbito de lo observable (las sustancias y sus propiedades); el "submicroscópico», que recurre a modelos corpusculares para interpretar la estructura de la materia; y el «simbólico», que permite comunicar y relacionar los fenómenos a escala macro o micro a través de símbolos, fórmulas o ecuaciones. 
Al igual que sucede con otras ideas centrales de la ciencia, el aprendizaje del cambio químico conlleva diversas dificultades que atañen a sus tres niveles de representación (González-Rodríguez y Crujeiras, 2016): distinguir los fenómenos físicos y químicos en base a indicios macroscópicos (Stavridou y Solomonidou, 1989), comprender la ordenación atómica después de la reacción o relacionar los niveles de representación submicroscópico y simbólico (Nurrenbern y Pickering, 1987), entre otras. Para abordar adecuadamente estas ideas y superar las dificultades asociadas, respaldamos un trabajo a través de actividades indagativas que promuevan el uso y evaluación de «modelos teóricos intermediarios» (Clement, 2000) y faciliten la construcción de un modelo global de cambio químico (Garrido, 2016).

En este trabajo, además de familiarizar al futuro profesorado de educación secundaria con las secuencias de problemas indagativos, se pretende promover su aprendizaje sobre un modelo escolar de cambio químico como el representado en la Tabla 1.

Tabla 1. Modelo de cambio químico para la Educación Secundaria Obligatoria (ESO)

\begin{tabular}{|l|l|}
\hline Nivel & Ideas \\
\hline Macroscópico & $\begin{array}{l}\text { La modificación de las propiedades características de las sustancias } \\
\text { (solubilidad, densidad, punto de fusión, etc.) indica que se ha producido } \\
\text { un cambio químico. }\end{array}$ \\
\hline Submicroscópico & $\begin{array}{l}\text { El cambio químico implica una reorganización de los átomos que } \\
\text { intervienen en el proceso, dando lugar a nuevas sustancias. Sin embargo, } \\
\text { el número y tipo de átomos se conservan, lo que justifica la conservación } \\
\text { de la masa. }\end{array}$ \\
\hline Simbólico & $\begin{array}{l}\text { La ecuación química es una representación simbólica del cambio químico, } \\
\text { donde intervienen reactivos y productos. La ecuación ajustada unifica } \\
\text { distintas "situaciones experimentales" asociadas a un mismo cambio } \\
\text { químico, "casos" en los que los reactivos limitante y excedente pueden ser } \\
\text { diferentes. }\end{array}$ \\
\hline
\end{tabular}

\section{Finalidad}

El trabajo forma parte de una investigación más amplia sobre la formación del futuro profesorado de física y química en el diseño e implementación de actividades escolares indagativas, a través de la Metodología de Resolución de Problemas como Investigación (MRPI). En este caso, se plantea el siguiente objetivo:

- Analizar los niveles de logro de futuros profesores de física y química en la resolución de problemas indagativos sobre los cambios físicos y químicos.

\section{Metodología}

\section{Muestra y contexto de la investigación}

El estudio se realiza en la asignatura de «Didáctica de la Química» del Máster en Formación del Profesorado de Secundaria (MFPS) de la Universidad Complutense de Madrid. En concreto, se desarrolla con un grupo-clase formado por 25 estudiantes (27,6 años de media; 10 químicos, 9 físicos y 6 estudiantes con otras titulaciones, sobre todo ingenierías).

La asignatura está organizada en torno a "problemas profesionales» (Martínez-Aznar et al., 2017), como p.ej.: ¿Qué deberían saber y ser capaces de hacer los profesores de física y química?; ¿Cómo se pueden seleccionar los contenidos para diseñar una Unidad Didáctica 
(UD)?; ¿Cómo se pueden evaluar las actividades...? Durante su resolución en grupos cooperativos -agrupados de modo que todos cuenten con físicos y químicos-, los formadores ofrecen "vivencias» de aprendizaje de corte innovador y promueven la reflexión sobre su propio rol de guías en el desarrollo de las actividades. El trabajo a lo largo de la asignatura se refleja en la elaboración y presentación de Unidades Didácticas (UD) escolares, donde el futuro profesorado debe defender sus concepciones y aprendizajes de carácter didáctico (Rodríguez-Arteche y Martínez-Aznar, 2018).

En particular, este artículo atañe al siguiente problema profesional: «¿Cómo se pueden diseñar e implementar actividades para una UD?». El problema se aborda en 7 sesiones de 1 hora y media a mitad del desarrollo de la asignatura (Martínez-Aznar et al., 2017) y, entre ellas, una parte importante se destina a la resolución por parte del futuro profesorado de una UD de corte indagativo propuesta por los formadores: "Cambio y Diversidad en la Naturaleza». Esta UD se ajusta a un nivel de 3 de ESO y está formada por una secuencia de cuatro situaciones problemáticas abiertas (Rodríguez-Arteche y MartínezAznar, 2016a, 2016c): ¿qué puede ocurrir...?

1. ... cuando dos sustancias se ponen en contacto?

2. ... cuando a una sustancia se le añade agua?

3. ... cuando se calienta una sustancia?

4. ... cuando una sustancia se pone en contacto con la corriente eléctrica?

El hecho de tratarse de problemas abiertos permite extender los objetivos de aprendizaje y ofrecer un andamiaje intencionadamente exigente para el futuro profesorado de secundaria. En todo caso, para facilitar el trabajo, los formadores proporcionan la "plantilla» de la MRPI mostrada en el Anexo 1, que recoge distintas etapas que pueden ser útiles en la resolución de los problemas. Las estrategias particulares de andamiaje -formulación de preguntas, modelización, retroalimentación, etc.-, así como los contenidos específicos de la propuesta didáctica pueden consultarse en Rodríguez-Arteche y Martínez-Aznar (2016a, 2016c).

\section{Recogida de los datos: problemas 1 y 3}

Se analizan los logros de los estudiantes en sus informes escritos finales para los problemas 1 y 3 -en los otros dos no se piden producciones escritas-. Estos informes se redactan ajustándose en la medida de lo posible a la «plantilla» de la MRPI (Anexo 1). Previamente, los futuros profesores llevan a cabo la resolución experimental de los problemas en grupos cooperativos, según se resume a continuación, pero para promover una reflexión personal se solicita que las producciones escritas sean individuales.

Al comienzo del problema 1 (Rodríguez-Arteche y Martínez-Aznar, 2016a), los grupos de futuros profesores realizan un análisis y diseñan estrategias que contemplan diversas sustancias a poner en contacto, en estados sólido, líquido o gaseoso. Sin embargo, los formadores acotan el problema proporcionando dos sustancias « $A$ » $y$ « $B$ » en estado sólido, desconocidas para el futuro profesorado (se trata de $\mathrm{Pb}\left(\mathrm{NO}_{3}\right)_{2}$ y KI). Esta elección promueve la indagación sobre posibles indicadores de una reacción química, dado que las sustancias iniciales -reactivos- son blancas y solubles en agua, pero uno de los productos $\left(\mathrm{PbI}_{2}\right)$ es amarillo e insoluble. El hecho de que el yoduro de potasio sea higroscópico también permite que la reacción se produzca a través del contacto en un mortero, lo que favorece la reflexión sobre factores cinéticos como la superficie de contacto o el tiempo. Además, según sean las cantidades añadidas, los participantes podrán determinar cuál de los dos reactivos es el excedente. Para ello, teniendo en cuenta que en el estado final la única sustancia insoluble es el yoduro de plomo (II), los participantes podrán filtrarlo, añadir partes alícuotas de los reactivos y observar en qué caso se vuelve a producir la reacción química. 
Para justificar las observaciones realizadas y relacionar los niveles simbólico y sub-microscópico del cambio químico (Nurrenbern y Pickering, 1987), se anima a que los participantes realicen representaciones microscópicas de lo sucedido a través de un modelo escolar adecuado (Windschitl et al., 2008), tras haber identificado las sustancias. Así, en los problemas P1-P3 el modelo atómico de Dalton resulta útil para representar "situaciones experimentales" específicas, considerando los estados de agregación, la conservación de la masa y la reorganización atómica en el proceso. Además, a partir de estas representaciones es posible obtener la ecuación química ajustada para la reacción, a través de un proceso que enfatiza la interpretación química y no la meramente matemática. Estos aspectos se representan en la Figura 2, que corresponde a un posible marco de referencia para analizar los logros de los participantes.
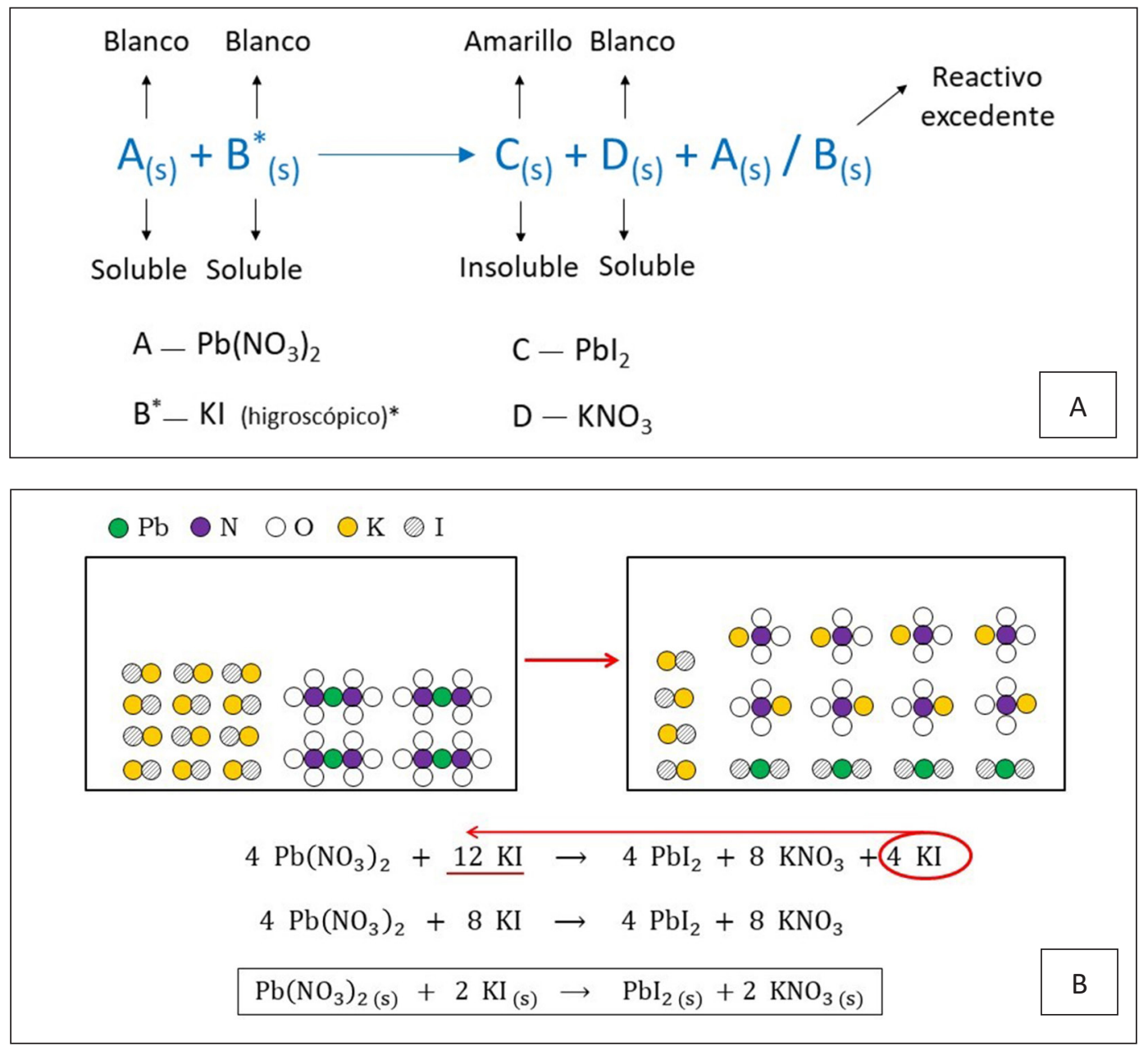

Figura 2. (A) Marco de referencia para la resolución del problema 1. (B) Representación microscópica (Modelo de Dalton) y ajuste de la ecuación química a partir de un "caso particular"

Al igual que antes, en su análisis inicial para el problema 3 (Rodríguez-Arteche y MartínezAznar, 2016c) los grupos de futuros profesores consideran distintos estados de agregación para la sustancia problema. No obstante, ya en el laboratorio la actividad se acota partiendo de una sustancia «C» sólida y desconocida (se trata de $\mathrm{KClO}_{3}$ ). El clorato de potasio presenta temperaturas de fusión y de descomposición térmica fácilmente alcanzables en los laboratorios escolares y, por ello, es una buena elección para explorar el criterio de la (ir)reversibilidad en relación con el tipo de cambio, y observar procesos de fusión/solidifi- 
cación y una reacción común de descomposición donde se obtiene un sólido a partir de un líquido. Además, el problema permite determinar que el gas emitido es oxígeno. Para ello, como parte de la labor de andamiaje, los formadores pueden introducir bolitas pequeñas de papel en los tubos de ensayo, lo que provoca combustiones espontáneas. Este fenómeno estimula el análisis y el debate en los grupos de participantes, así como los procesos de argumentación (Jiménez-Aleixandre y Puig, 2012). Nuevamente, se promueve la representación microscópica de los procesos de cambio y el análisis global de los resultados, haciendo referencia a las hipótesis. En este caso, un posible marco de referencia para comprender los logros de los participantes es el de la Figura 3.

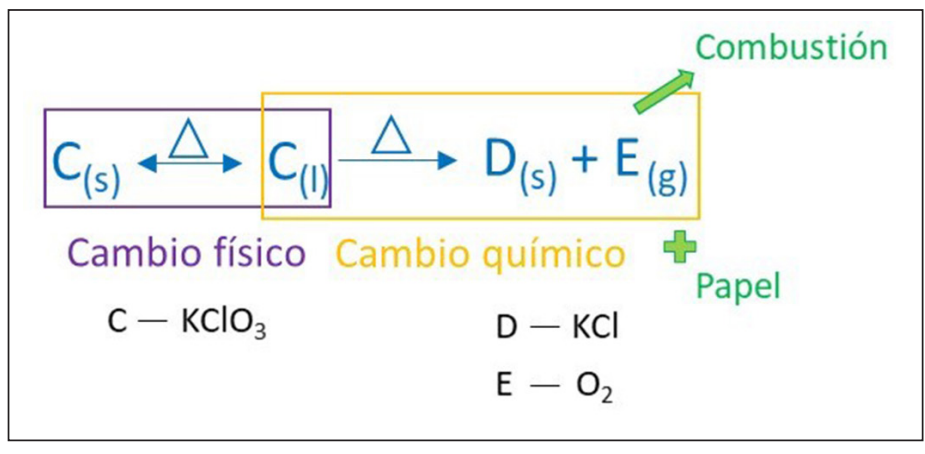

Figura 3. Marco de referencia para la resolución del problema 3

Como inciso, indicar que en esta UD orientada a la Educación Secundaria Obligatoria, se asume la transposición didáctica de considerar los cambios químicos como procesos irreversibles -los ejemplos proporcionados se ajustan a esta decisión-. El equilibrio químico requeriría de otro tipo de secuencias didácticas (Raviolo y Martínez-Aznar, 2003).

\section{Análisis de los datos}

Para el análisis de los datos, es decir, de los informes individuales sobre los problemas 1 y 3 , se toman como referencia las cinco fases o Dimensiones de la Competencia científica (DC) incluidas en la MRPI. Asimismo, las etapas 1 y 3 se subdividen como:

- DC1.1. Representación cualitativa del problema (marco teórico)

- DC1.2. Reformulación del problema

- DC3.1. Identificación y control de variables

- DC3.2. Toma de decisiones para el problema

En cada caso, se definen niveles de logro desde 0 (irrelevante) hasta 4 (muy satisfactorio), en función de la información contenida y de la presencia de errores conceptuales y procedimentales. El Anexo 2 contiene las descripciones precisas de estos niveles de logro para las distintas dimensiones competenciales (DC). Estas definiciones son fruto de la discusión conjunta y el consenso entre los autores del artículo, precedidos de la revisión de otros trabajos de la literatura sobre la evaluación de la indagación (Ferrés, Marbà y Sanmtartí, 2015; Franco-Mariscal, 2015).

Para conocer el éxito en las dimensiones competenciales (en términos comparativos), se calculan los porcentajes (\%) de futuros profesores en cada nivel de resolución, así como los Niveles Medios (NM) de logro, de forma similar a otros estudios (Martínez-Aznar y Varela, 2009; Rodríguez-Arteche et al., 2016). Siguiendo una línea más descriptiva, el trabajo también expone ejemplos extraídos de los informes de los futuros profesores y representa varios de sus análisis en diagramas de Toulmin (1958). 


\section{Resultados}

A partir de los informes finales de los futuros profesores, se identifican los niveles para las dimensiones competenciales (DC) en ambos problemas: P1. «¿Qué puede ocurrir cuando dos sustancias se ponen en contacto?» y P3. "¿Qué puede ocurrir cuando se calienta una sustancia?», resueltos a través de la MRPI. La Tabla 2 contiene los porcentajes de estudiantes del Máster situados en cada uno de los niveles de resolución (N0-N4), los niveles medios de logro (NM) y las medianas para las dimensiones competenciales (con sombreados). Cabe indicar que, en los momentos establecidos para la recogida de las producciones escritas, el Informe 1 lo entregan 17 futuros profesores, mientras que el Informe 2 es remitido por 24 participantes.

Tabla 2. Distribución de las dimensiones competenciales en niveles de logro (\%), niveles medios (NM) y medianas (con sombreado), en ambos problemas abiertos

\begin{tabular}{|l|r|r|r|r|r|r|r|r|r|r|r|l|}
\hline Dimensión & N0 & N1 & N2 & N3 & N4 & NM & N0 & N1 & N2 & N3 & N4 & NM \\
\hline DC1.1 Repres. cualitativa & 0 & 41 & 18 & 29 & 12 & $\mathbf{2 , 1 2}$ & 0 & 8 & 33 & 33 & 25 & $\mathbf{2}, 75$ \\
\hline DC1.2 Reformulación & 18 & 12 & 18 & 24 & 29 & $\mathbf{2 , 3 5}$ & 0 & 4 & 54 & 13 & 29 & $\mathbf{2 , 6 7}$ \\
\hline DC2 Emisión de hipótesis & 0 & 6 & 24 & 24 & 47 & $\mathbf{3 , 1 2}$ & 0 & 0 & 29 & 13 & 58 & 3,29 \\
\hline DC3.1 Identif. variables & 6 & 41 & 12 & 24 & 18 & $\mathbf{2 , 0 6}$ & 0 & 21 & 46 & 17 & 17 & $\mathbf{2 , 2 9}$ \\
\hline DC3.2 Toma de decisiones & 6 & 24 & 41 & 24 & 6 & $\mathbf{2 , 0 0}$ & 0 & 21 & 33 & 29 & 17 & $\mathbf{2 , 4 2}$ \\
\hline DC4 Resolución experim. & 0 & 12 & 35 & 41 & 12 & $\mathbf{2 , 5 3}$ & 0 & 13 & 25 & 42 & 21 & $\mathbf{2 , 7 1}$ \\
\hline DC5 Análisis de resultados & 0 & 29 & 35 & 35 & 0 & $\mathbf{2 , 0 6}$ & 0 & 8 & 29 & 33 & 29 & $\mathbf{2 , 8 3}$ \\
\hline
\end{tabular}

*Los indicadores donde hay mejoría al resolver el segundo problema están en verde, y el caso que obtiene un resultado peor está en rojo

Según se observa en la Tabla 2, todas las dimensiones competenciales obtienen medianas y niveles medios de logro iguales o superiores a 2 en ambos problemas, aunque existen diferencias apreciables entre estas destrezas. Si atendemos a las medianas, las capacidades como la representación cualitativa de los problemas (DC1.1), la emisión de hipótesis (DC2) o el análisis final de resultados (DC5) mejoran de forma relevante en el proceso, pero en el caso de la reformulación de los enunciados en términos operativos (DC1.2) detectamos un retroceso. En cambio, si nos fijamos en las medias, todas las dimensiones progresan a niveles de mayor complejidad. Esto último es visible en la Figura 4.

La figura muestra que, en el conjunto de los dos problemas los mejores resultados se obtienen en la emisión de hipótesis (DC2) y en la fase de resolución del problema (DC4). Lo primero podría explicarse por la relación de estas actividades con conceptos estructurantes de la química, un aspecto sugerido como promotor del éxito en las prácticas científicas (Kang et al., 2016). Así, se formula un buen número de hipótesis clasificadas en los niveles superiores, como p.ej.: "si asumimos que la transferencia de energía puede promover reacciones de combinación $(A+B \rightarrow A B)$ y descomposición $(A B \rightarrow A+B)$, al calentar una sustancia se producirá un cambio químico (P3, Nivel 4)». Por otra parte, los buenos resultados en la DC4 podrían justificarse atendiendo a la familiarización de los futuros profesores con los componentes de esta dimensión competencial: describir procedimientos y observaciones, registrar y tratar datos diversos, etc. -elementos siempre incluidos en los Grados en Química o Física- (Madsen, McKagan y Sayre, 2015). 


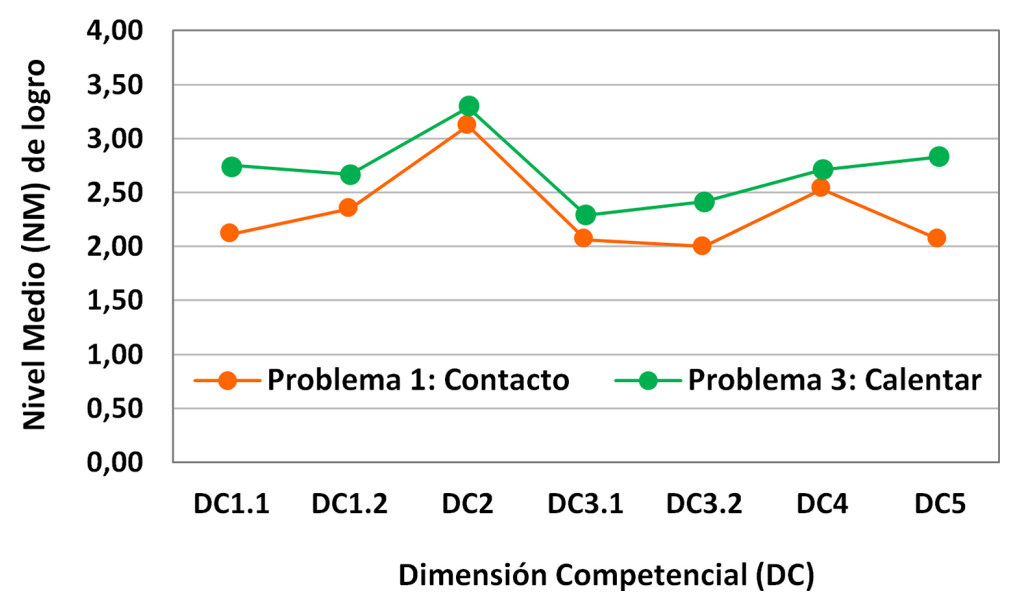

Figura 4. Resultados en las dimensiones competenciales para los problemas abiertos 1 y 3

Los peores resultados de la figura corresponden a la fase 3 de la MRPI, que abarca la identificación y control de las variables de los problemas (DC3.1) y la toma de decisiones para resolverlos (DC3.2). Estas dificultades, también detectadas en otros trabajos en torno a la indagación (Ferrés, Marbà y Sanmartí, 2015; García-Carmona et al., 2017), guardan una semejanza con la forma tradicional de plantear la actividad experimental en los niveles escolares y universitarios - muy pautada, a través de guiones cerrados- y con la escasa autonomía otorgada a los estudiantes.

La Tabla 3 incluye ejemplos asignados a distintos niveles de logro en la DC3.1 (identificación y control de variables) para el primer problema. Los estudiantes que obtienen un nivel 2 identifican una variable dependiente adecuada y aquellos con un nivel 3 también distinguen una independiente. El nivel 4 conlleva explicitar algunas variables de control.

Tabla 3. Clasificación de respuestas para la DC3.1 en el problema abierto «¿Qué puede ocurrir cuando dos sustancias se ponen en contacto?»

\begin{tabular}{|c|c|}
\hline $\begin{array}{l}\text { Nivel } \\
\text { (FP) }\end{array}$ & Ejemplo de respuesta de los futuros profesores (FP) \\
\hline $\begin{array}{c}1 \\
(F P 16)\end{array}$ & $\begin{array}{l}\text { VI: composición de los reactivos } \\
\text { VD: transformaciones químicas, grado de mezcla, estado de agregación de la } \\
\text { materia } \\
\text { VC: temperatura y presión atmosférica }\end{array}$ \\
\hline $\begin{array}{c}2 \\
(F P \text { 13) }\end{array}$ & $\begin{array}{l}\text { VI: temperatura } \\
\text { VD: reactividad de A con B (reaccionan o no reaccionan) } \\
\text { VC: presión atmosférica }\end{array}$ \\
\hline $\begin{array}{c}3 \\
(F P \text { 14) }\end{array}$ & $\begin{array}{l}\text { VI: tipo de sustancias (su naturaleza y propiedades) } \\
\text { VD: tipo de reacción que sucede (reacción química) }\end{array}$ \\
\hline $\begin{array}{c}4 \\
\text { (FP 12) }\end{array}$ & $\begin{array}{l}\text { VI: tipo de sustancias } \\
\text { VD: cambio físico (hipótesis 1) o cambio químico (hipótesis 2) } \\
\text { VC: temperatura }\end{array}$ \\
\hline
\end{tabular}

*VI: variable independiente; VD: variable dependiente; VC: variables de control 
En relación con la evolución de los participantes, la mejoría más reseñable sucede en la representación cualitativa de los problemas (DC1.1) y en el análisis de resultados (DC5). Conviene poner en valor estos datos, ya que una de las críticas asociadas a las estrategias indagativas es la del predominio de los procedimientos científicos sobre otras capacidades como la construcción de marcos teóricos, el uso de modelos o la evaluación de explicaciones científicas. Precisamente, estos son los aspectos donde se detecta una mejoría mayor (Figura 4), probablemente fomentada por la discusión grupal del primer problema abierto y la revisión de los informes por los formadores (Zembal-Saul et al., 2000).

Para comprender mejor los aspectos descritos en los marcos teóricos (DC1.1) de los futuros profesores, según su nivel de logro, la Tabla 4 muestra los conceptos incluidos en las producciones escritas de cuatro futuros profesores para el problema "¿Qué puede ocurrir cuando se calienta una sustancia?». En coherencia con el Anexo 2, la tabla refleja que lo que marca la diferencia entre los niveles superiores e inferiores es el desarrollo de las nociones de cambio físico y químico -con sus distintos niveles de representación-, ya que este es el contenido central del problema abierto. También se percibe la variedad de conceptos justificados en los informes del futuro profesorado (el $58 \%$ de ellos se sitúa en N3 o N4 en el problema final), aunque se echa en falta el análisis previo de las "posibilidades" de cambio físico o químico según sea el estado de agregación de "C» (es decir, antes de acotar el problema con $\mathrm{KClO}_{3}$ en estado sólido).

Tabla 4. Clasificación de respuestas para la DC1.1 en el problema abierto «¿Qué puede ocurrir cuando se calienta una sustancia?», a partir de los conceptos descritos en el marco teórico

\begin{tabular}{|l|c|c|c|c|c|}
\cline { 2 - 6 } \multicolumn{1}{c|}{} & \multicolumn{5}{c|}{ Niveles de logro obtenidos por los } \\
\hline \multicolumn{1}{|c|}{ Conceptos incluidos en el marco teórico } & $\begin{array}{c}\text { N1 } \\
\text { (FP 10) }\end{array}$ & $\begin{array}{c}\text { N2 } \\
\text { (FP 14) }\end{array}$ & $\begin{array}{c}\text { N3 } \\
\text { (FP 23) }\end{array}$ & $\begin{array}{c}\text { N4 } \\
\text { (FP 1) }\end{array}$ \\
\hline Materia & $\checkmark$ & & $\checkmark$ & $\checkmark$ \\
\hline Sustancia química & $\checkmark$ & $\checkmark$ & $\checkmark$ & $\checkmark$ \\
\hline Elemento & $\checkmark$ & & $\checkmark$ & $\checkmark$ \\
\hline Compuesto & $\checkmark$ & & $\checkmark$ & $\checkmark$ \\
\hline Propiedades de las sustancias (extensivas/intensivas) & $\checkmark$ & & $\checkmark$ & $\checkmark$ \\
\hline Estados de agregación & & $\checkmark$ & $\checkmark$ & $\checkmark$ \\
\hline Temperatura & & $\checkmark$ & $\checkmark$ & $\checkmark$ \\
\hline Calor & $\checkmark$ & $\checkmark$ & $\checkmark$ & $\checkmark$ \\
\hline Cambio físico: & & $\checkmark$ & $\checkmark$ & $\checkmark$ \\
\hline Características generales & $\checkmark$ & $\checkmark$ & $\checkmark$ & $\checkmark$ \\
\hline Cambios de estado & & & & \\
\hline Cambio químico/reacción: & & $\checkmark$ & $\checkmark$ & $\checkmark$ \\
\hline Cambio en las propiedades (nivel macroscópico) & & & $\checkmark$ & $\checkmark$ \\
\hline Reordenación atómica (nivel submicroscópico) & & & & $\checkmark$ \\
\hline Ecuación química (nivel simbólico) & & & & $\checkmark$ \\
\hline $\begin{array}{l}\text { Reacciones químicas en el contexto del problema: } \\
\text { descomposición, combustión, oxidación, etc. }\end{array}$ & $\checkmark$ & & & $\checkmark$ & $\checkmark$ \\
\hline Reversibilidad/irreversibilidad de los cambios & & $\checkmark$ & $\checkmark$ & & $\checkmark$ \\
\hline Ley de conservación de la masa & & & & & \\
\hline Posibilidades en función del estado de agregación de C & & & & \\
\hline
\end{tabular}




\section{Los procesos de argumentación en la resolución de los problemas abiertos}

Como análisis complementario, se presenta una síntesis de los procesos de argumentación desarrollados en varias producciones categorizadas con niveles de logro 3 o 4 en todas las dimensiones competenciales -se examina un informe por cada problema abierto-. Para ello, los autores del artículo representamos las relaciones establecidas entre datos, hipótesis, marcos teóricos y conclusiones a través de diagramas de Toulmin (1958), centrándonos en los aspectos citados (Crujeiras y Jiménez-Aleixandre, 2015).

La Figura 5 representa los procesos argumentativos realizados por un futuro profesor en su informe sobre «¿Qué puede ocurrir cuando dos sustancias se ponen en contacto?». Para simplificar el diagrama, la figura omite ciertos aspectos de la producción escrita, como la determinación del reactivo limitante.

\begin{tabular}{|c|c|c|c|c|c|}
\hline \multirow{3}{*}{\multicolumn{2}{|c|}{$\begin{array}{l}\text { ¿Qué puede ocurrir cuando } \\
\text { dos sustancias se ponen en } \\
\text { contacto? }\end{array}$}} & \multirow{2}{*}{\multicolumn{2}{|c|}{$\begin{array}{l}\text { Un cambio significativo en una prop. } \\
\text { caracteristica (p.ej., la solubilidad), } \\
\text { informa de un cambio en la naturaleza } \\
\text { de las sustancias (reorganización } \\
\text { atómica) } \rightarrow \text { Relación entre niveles } \\
\text { macroscópico y microscópico. }\end{array}$}} & \multirow{3}{*}{\multicolumn{2}{|c|}{$\begin{array}{l}\text { RESPALDO } \\
\text { Marco Teórico } \\
\text { *Se asume la transposición did } \\
\text { tica de tomar las reacciones co } \\
\text { procesos irreversibles. }\end{array}$}} \\
\hline & & & & & \\
\hline & & \multicolumn{2}{|c|}{ debido a } & & \\
\hline \multicolumn{2}{|l|}{ DATOS } & \multirow{2}{*}{\multicolumn{2}{|c|}{$\begin{array}{l}\text { El contacto provoca la formación de } \\
\text { nuevas sustancias. }\end{array}$}} & JUSTIFICACIÓN & CONCLUS \\
\hline \multirow{3}{*}{\multicolumn{2}{|c|}{$\begin{array}{l}\text { - El contacto en estado sólido } \\
\text { (con mortero) da lugar a un } \\
\text { cambio de color del sistema, } \\
\text { de blanco a amarillo. } \\
\text { - Tras el contacto, se produce } \\
\text { un cambio significativo en la } \\
\text { solubilidad de las sustancias. }\end{array}$}} & & & \multirow{3}{*}{\multicolumn{2}{|c|}{$\begin{array}{l}\text { Se corrobora la hipótesis de } \\
\text { cambio químico: del cambio a } \\
\text { nivel macroscópico (solubilidad) } \\
\text { se concluye un cambio en la } \\
\text { reorganización atómica: } \\
A_{(s)}+B_{(s)} \rightarrow C_{(s)}+D_{(s)} *\end{array}$}} \\
\hline & & \multicolumn{2}{|c|}{ ya que } & & \\
\hline & & \multicolumn{2}{|r|}{ Por tanto } & & \\
\hline DUDAS & \multicolumn{2}{|c|}{$\begin{array}{l}\text { ¿Qué implica el cambio de } \\
\text { color? }\end{array}$} & \multicolumn{3}{|c|}{\begin{tabular}{|l|} 
¿Cómo es posible un cambio en estado sólido? \\
¿Equivale al caso de poner en contacto disoluciones?
\end{tabular}} \\
\hline Marco Teórico & \multicolumn{2}{|c|}{$\begin{array}{l}\text { ¿El color puede ser un criterio } \\
\text { para diferenciar los cambios } \\
\text { fisicos y químicos? }\end{array}$} & \multicolumn{3}{|c|}{$\begin{array}{l}\text { ¿En qué estados de agregación pueden encontrarse } \\
\text { los reactivos? ¿Qué implicaciones cinéticas conlleva? }\end{array}$} \\
\hline
\end{tabular}

Figura 5. Diagrama de Toulmin que refleja el análisis de un futuro profesor para el Problema 1

Según se observa, a lo largo del informe el futuro profesor recoge y justifica datos (DC4) para dar respuesta a la pregunta de investigación a través de conclusiones (DC5) y, para ello, se apoya en el marco teórico construido (DC1.1). Así, el sujeto argumenta a partir del criterio de las propiedades características (cambio significativo en la solubilidad $\Rightarrow$ cambio químico). Además, su informe describe ciertas dudas sobre la relación del color con los cambios físicos y químicos, o acerca de los estados de agregación en que suceden las reacciones. Estos aspectos sugieren la conveniencia de diseñar secuencias de problemas indagativos -como en esta propuesta-, para permitir la evaluación y reconstrucción progresiva de los marcos teóricos o modelos propuestos (Hernández et al., 2015; Kang et al., 2016).

La Figura 6 corresponde a un diagrama equivalente para el análisis -satisfactorio- realizado por otro futuro profesor en su producción escrita sobre «¿Qué puede ocurrir cuando se calienta una sustancia?». Por simplicidad, no se han incluido los datos y argumentos aportados para una primera etapa de fusión de la sustancia.

Al igual que en el ejemplo anterior, en este caso (Figura 6) el estudiante del Máster realiza un análisis muy completo amparado en conceptos como la (ir)reversibilidad de los 
cambios, la temperatura de fusión o las condiciones para que se produzca una reacción de combustión. Ello le permite concluir que se ha producido un cambio químico -descomposición- que indica que la sustancia aportada en la actividad es un compuesto.

En general, se constata que secuencias de problemas abiertos como las mostradas, con un andamiaje cuidadosamente planificado (Rodríguez-Arteche y Martínez-Aznar, 2016a), favorecen un trabajo relevante sobre las destrezas indagativas $y$, además, sobre los conceptos químicos.

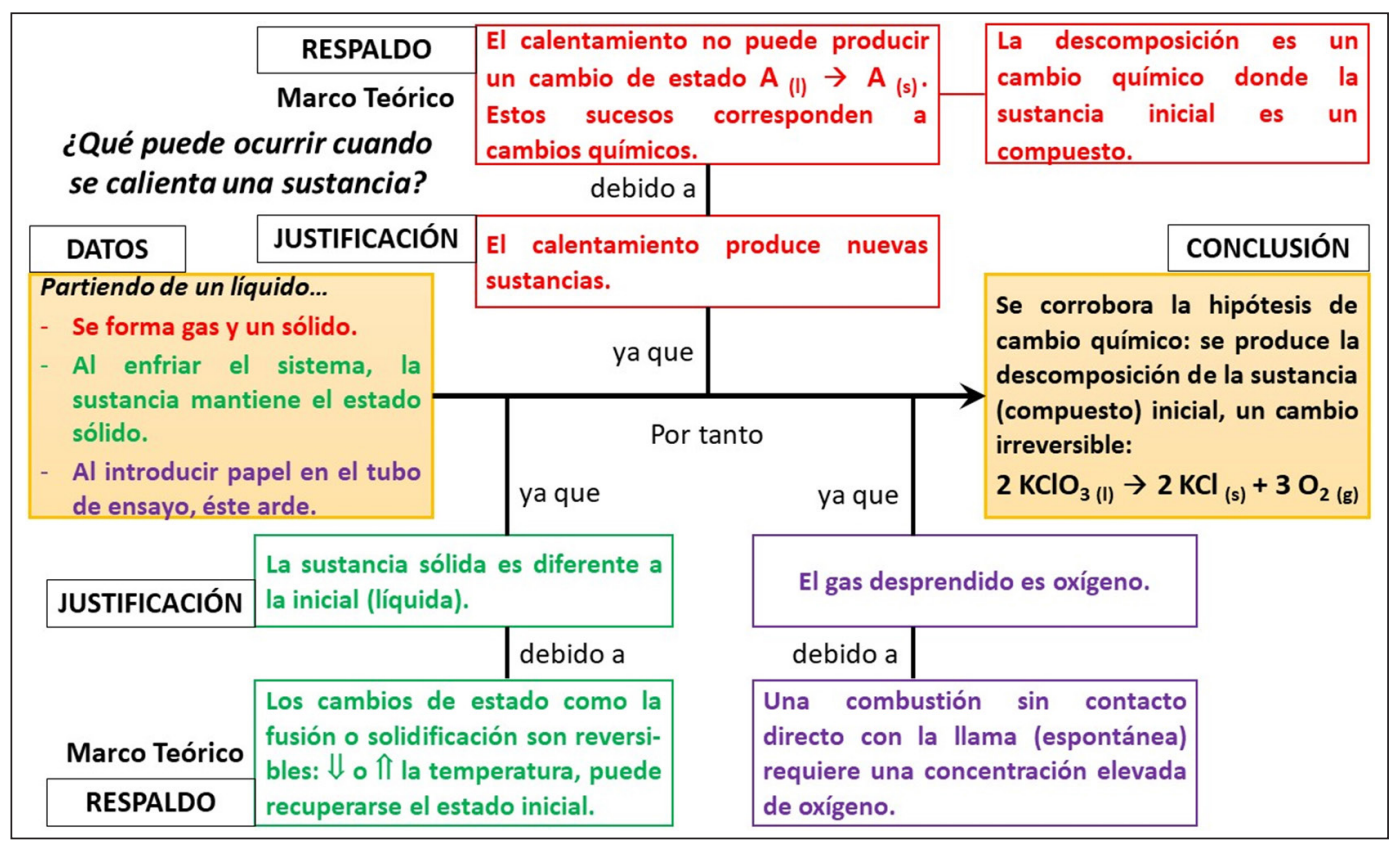

Figura 6. Diagrama de Toulmin que refleja el análisis de un futuro profesor para el Problema 3

\section{Conclusiones}

El análisis realizado ha permitido comprobar el desempeño y la mejoría del futuro profesorado en las dimensiones de la competencia científica incluidas en la MRPI, la metodología indagativa abordada en la asignatura de Didáctica de la Química. Así, tras una evolución especialmente acusada en las dimensiones más conceptuales (DC1.1, Representación cualitativa del problema y DC5, Análisis de resultados), la mayor parte de las DC se acercan a un nivel medio de logro cercano a 3 , un resultado positivo. La excepción corresponde a la fase del Diseño de estrategias de resolución (DC3.1, DC3.2), lo que sugiere que los estudiantes del Máster, a pesar de su formación, encuentran difícil decidir por sí mismos los criterios y estrategias para resolver problemas abiertos de tipo experimental. Estos datos suponen un estímulo para cambiar la forma tradicional de abordar el trabajo de laboratorio-actividades tipo «receta»- en los niveles escolares y universitarios.

Por otra parte, el artículo aporta materiales que pueden ser de utilidad en la formación docente y para el aprendizaje de los escolares de secundaria. En este sentido, creemos que propuestas formativas como la actual pueden ser de gran valor para promover la reflexión del futuro profesorado sobre los beneficios de la indagación (Rodríguez-Arteche y Martínez-Aznar, 2016b), no solo como «fin» en sí misma, sino como «medio» para facilitar un mejor aprendizaje conceptual (Barrow, 2006). En esta línea, la secuencia indagativa presentada está ligada a la construcción de un modelo escolar de cambio químico, 
y conlleva el trabajo sobre una gran variedad de aspectos asociados a sus tres niveles de representación. Por ello, mantenemos que contraponer este tipo de actividades con otros enfoques de enseñanza-aprendizaje (Jiménez-Tenorio y Oliva, 2016), en el marco de la resolución de un "problema profesional» (Martínez-Aznar et al., 2017), supone una tarea que, desde la formación inicial del profesorado de educación secundaria, estamos obligados a plantear.

No obstante, cabe tener presentes ciertas limitaciones del estudio, como el tamaño de la muestra o la obtención de resultados solamente a través de los informes escritos. Por ello, en futuros trabajos pretendemos incidir en la construcción del modelo de cambio químico a través de registros en vídeo y entrevistas grupales. Además, el estudio realizado abre nuevas líneas de investigación acerca del uso de la indagación en los comienzos docentes. Algunas de las preguntas que cabe plantearse son: ¿Hasta qué punto implementan los egresados del Máster las secuencias de problemas de tipo indagativo? ¿Qué tipo de andamiaje proporcionan? ¿Qué criterios utilizan para diseñar y/o seleccionar propuestas didácticas en esta línea? En próximos trabajos pretendemos contribuir al análisis sobre estas y otras cuestiones.

\section{Referencias bibliográficas}

Abd-El-Khalick, F., BouJaoude, S., Duschl, R., Lederman, N.G., Mamlok-Naaman, R., Hofstein, A., Niaz, M., Treagust, D. y Tuan, H-L. (2004). Inquiry in Science Education: International Perspectives. Science Education, 88(3), 397-419.

Acevedo, J.A., García-Carmona, A., Aragón, M.M. y Oliva, J.M. (2017). Modelos científicos: significado y papel en la práctica científica. Revista Científica, 30(3), 155-166.

Aguilera, D., Martín-Paez, T., Valdivia, V., Ruiz-Delgado, Á., Williams, L., Vílchez, J.M. y Perales, F.J. (2018). La enseñanza de las ciencias basada en indagación. Una revisión sistemática de la producción española. Revista de Educación, 381, 259-284.

Aragón, M.M., Oliva, J.M. y Navarrete, A. (2013). Evolución de los modelos explicativos de los alumnos en torno al cambio químico a través de una propuesta didáctica con analogías. Enseñanza de las Ciencias, 31(2), 9-30.

Barrow, L.H. (2006). A Brief History of Inquiry: From Dewey to Standards. Journal of Science Teacher Education, 17(3), 265-278.

Bevins, S. y Price, G. (2016). Reconceptualising inquiry in science education. International Journal of Science Education, 38(1), 17-29.

Bybee, R.W., Taylor, J.A., Gardner, A., Van Scotter, P., Powell, J.C., Westbrook, A. y Landes, N. (2006). The BSCS 5E Instructional Model: Origins, Effectiveness, and Applications. Colorado Springs, Co: BSCS.

Capps, D.K. y Crawford, B.A. (2013). Inquiry-Based Instruction and Teaching About Nature of Science: Are They Happening? Journal of Science Teacher Education, 24(3), 497-526.

Clement, J. (2000). Model Based Learning as a Key Research Area for Science Education. International Journal of Science Education, 22(9), 1041-1053.

Cobern, W.W., Schuster, D., Adams, B., Applegate, B., Skjold, B., Undreiu, A., Loving, C.C. y Gobert, J.D. (2010). Experimental comparison of inquiry and direct instruction in science. Research in Science \& Technological Education, 28(1), 81-96. 
Crawford, B.A. (2007). Learning to Teach Science as Inquiry in the Rough and Tumble of Practice. Journal of Research in Science Teaching, 44(4), 613-642.

Crujeiras, B. (2017). Análisis de las estrategias de apoyo elaboradas por futuros docentes de educación secundaria para guiar al alumnado en la indagación. Revista Eureka sobre Enseñanza y Divulgación de las Ciencias, 14(2), 473-486.

Crujeiras, B. y Jiménez-Aleixandre, M.P. (2015). Desafíos planteados por las actividades abiertas de indagación en el laboratorio: articulación de conocimientos teóricos y prácticos en las prácticas epistémicas. Enseñanza de las Ciencias, 33(1), 63-84.

Domènech, J. (2013). Secuencias de apertura experimental y escritura de artículos en el laboratorio: Un itinerario de mejora de los trabajos prácticos en el laboratorio. Enseñanza de las Ciencias, 31(3), 249-262.

Ferrés, C., Marbà, A. y Sanmartí, N. (2015). Trabajos de indagación de los alumnos: instrumentos de evaluación e identificación de dificultades. Revista Eureka sobre Enseñanza y Divulgación de las Ciencias, 12(1), 22-37.

Franco-Mariscal, A.J. (2015). Competencias científicas en la enseñanza y el aprendizaje por investigación. Un estudio de caso sobre corrosión de metales en secundaria. Enseñanza de las Ciencias, 33(2), 231-352.

García-Carmona, A., Criado, A.M. y Cruz-Guzmán, M. (2017). Primary pre-service teachers' skills in planning a guided scientific inquiry. Research in Science Education, 47(5), 989-1010.

Garrido, A. (2016). Modelització i models en la formació inicial de mestres de primària des de la perspectiva de la pràctica científica. Tesis doctoral. Bellaterra: Universitat Autònoma de Barcelona.

Gil-Pérez, D. y Martínez-Torregrosa, J. (1983). A model for problem-solving in accordance with scientific methodology. European Journal of Science Education, 5(4), 447-455.

Gil-Quílez, M.J., Martínez-Peña, M.B. y Cordero, S. (2017). Grabaciones de situaciones de aula para la formación del profesorado. Ápice. Revista de Educación Científica, 1(1), 58-73. DOI: https://doi.org/10.17979/arec.2017.1.1.2005

González-Rodríguez, L. y Crujeiras, B. (2016). Aprendizaje de las reacciones químicas a través de actividades de indagación en el laboratorio sobre cuestiones de la vida cotidiana. Enseñanza de las Ciencias, 34(3), 143-160.

Harlen, W., Bell, D., Devés, R., Dyasi, H., Fernández, G., Léna, P., ..., Yu, W. (2015). Working with Big Ideas of Science Education. Trieste: Science Education Programme (SEP) of IAP.

Hernández, M.I., Couso, D. y Pintó, R. (2015). Analyzing Students' Learning Progressions Throughout a Teaching Sequence on Acoustic Properties of Materials with a ModelBased Inquiry Approach. Journal of Science Education and Technology, 24(2-3), 356-377.

Hmelo-Silver, C.E., Duncan, R.G. y Chinn, C.A. (2007). Scaffolding and Achievement in Problem-Based and Inquiry Learning: A Response to Kirschner, Sweller, and Clark (2006). Educational Psychologist, 42(2), 99-107.

Hung, W. (2016). All PBL Starts Here: The Problem. Interdisciplinary Journal of ProblemBased Learning, 10(2), article 2. 
Ibáñez, T. y Martínez-Aznar, M.M. (2005). Solving Problems in Genetics II: Conceptual restructuring. International Journal of Science Education, 27(12), 1495-1519.

Ibáñez, T. y Martínez-Aznar, M.M. (2007). Solving Problems in Genetics, Part III: Change in the view of the nature of science. International Journal of Science Education, 29(6), 747-769.

Jiménez-Aleixandre, M.P. y Puig, B. (2012). Argumentation, Evidence Evaluation and Critical Thinking. En B. Fraser, K. Tobin y C. McRobbie (Eds.), Second International Handbook of Science Education (pp. 1001-1015). Dordretch: Springer.

Jiménez-Tenorio, N. y Oliva, J.M. (2016). Aproximación al estudio de las estrategias didácticas en ciencias experimentales en formación inicial del profesorado de Educación Secundaria: descripción de una experiencia. Revista Eureka sobre Enseñanza y Divulgación de las Ciencias, 13(1), 121-136.

Johnstone, A.H. (1982). Macro- and micro-chemistry. School Science Review, 64, 377-379.

Kang, H., Windschitl, M., Stroupe, D. y Thompson, J. (2016). Designing, Launching, and Implementing High Quality Learning Opportunities for Students that Advance Scientific Thinking. Journal of Research in Science Teaching, 53(9), 1316-1340.

Kirschner, P.A., Sweller, J. y Clark, R.E. (2006). Why Minimal Guidance During Instruction Does Not Work: An Analysis of the Failure of Constructivist, Discovery, ProblemBased, Experiential, and Inquiry-Based Teaching. Educational Psychologist, 41(2), 75-86.

Madsen, A., McKagan, S.B. y Sayre, E.C. (2015). How physics instruction impacts students' beliefs about learning physics: A meta-analysis of 24 studies. Physical Review, Physics Education Research, 11(1), 10115.

Martín del Pozo, R. (2001). Lo que saben y lo que pretenden enseñar los futuros profesores sobre el cambio químico. Enseñanza de las Ciencias, 19(2), 199-215.

Martínez-Aznar, M.M. y Bárcena, A.I. (2013). Una actividad de indagación en un aula de diversificación: «¿Es beneficioso masticar bien para realizar una buena digestión?». Educació Química, EduQ, 14, 19-28.

Martínez-Aznar, M.M., Rodríguez-Arteche, I. y Gómez-Lesarri, P. (2017). La resolución de problemas profesionales como referente para la formación inicial del profesorado de física y química. Revista Eureka sobre Enseñanza y Divulgación de las Ciencias, 14(1), 162-180.

Martínez-Aznar, M.M. y Varela, P. (2009). La resolución de problemas de energía en la formación inicial de maestros. Enseñanza de las Ciencias, 27(3), 343-360.

Martínez-Chico, M., Jiménez-Liso, M.R. y López-Gay, R. (2015). Efecto de un programa formativo para enseñar ciencias por indagación basada en modelos, en las concepciones didácticas de los futuros maestros. Revista Eureka sobre Enseñanza y Divulgación de las Ciencias, 12(1), 149-166.

Nurrenbern, S.C. y Pickering, M. (1987). Concept learning versus problem solving: Is there a difference? Journal of Chemical Education, 64(6), 508-510.

Pilitsis, V. y Duncan, R.G. (2012). Changes in Belief Orientations of Preservice Teachers and Their Relation to Inquiry Activities. Journal of Science Teacher Education, 23(8), 909-936. 
Prince, M.J. y Felder, R.M. (2006). Inductive Teaching and Learning Methods: Definitions, Comparisons, and Research Bases. Journal of Engineering Education, 95(2), 123-138.

Raviolo, A. y Martínez-Aznar, M.M. (2003). Una revisión sobre las concepciones alternativas de los estudiantes en relación con el equilibrio químico. Clasificación y síntesis de sugerencias didácticas. Educación Química, 14(3), 159-165.

Reiser, B.J. (2004). Scaffolding Complex Learning: The Mechanisms of Structuring and Problematizing Student Work. Journal of the Learning Sciences, 13(3), 273-304.

Rodríguez-Arteche, I. y Martínez-Aznar, M.M. (2016a). Introducing Inquiry-Based Methodologies during Initial Secondary Education Teacher Training Using an OpenEnded Problem about Chemical Change. Journal of Chemical Education, 93(9), 1528-1535.

Rodríguez-Arteche, I. y Martínez-Aznar, M.M. (2016b). Indagación y modelos didácticos: La reflexión de cuatro profesores de física y química en formación inicial. Campo Abierto, 35(1), 145-160.

Rodríguez-Arteche, I. y Martínez-Aznar, M.M. (2016c). Open-ended problem solving in chemistry during initial secondary education teacher training. International Journal of Learning and Teaching, 8(3), 174-186.

Rodríguez-Arteche, I. y Martínez-Aznar, M.M. (2018). Evaluación de una propuesta para la formación inicial del profesorado de Física y Química a través del cambio en las creencias de los participantes. Revista Eureka sobre Enseñanza y Divulgación de las Ciencias, 15(1), 1601.

Rodríguez-Arteche, I., Martínez-Aznar, M.M. y Garitagoitia, A. (2016). La competencia sobre planificación de investigaciones en 40 de ESO: un estudio de caso. Revista Complutense de Educación, 27(1), 329-351.

Romero-Ariza, M. (2017). El aprendizaje por indagación: ¿existen suficientes evidencias sobre sus beneficios en la enseñanza de las ciencias? Revista Eureka sobre Enseñanza y Divulgación de las Ciencias, 14(2), 286-299.

Stavridou, H. y Solomonidou, C. (1989). Physical phenomena - chemical phenomena: do pupils make the distinction? International Journal of Science Education, 11(1), 83-92.

Toma, R.B., Greca, I.M. y Meneses, J.A. (2017). Dificultades de maestros en formación inicial para diseñar unidades didácticas usando la metodología de indagación. Revista Eureka sobre Enseñanza y Divulgación de las Ciencias, 14(2), 442-457.

Toulmin, S. (1958). The Uses of Argument. Cambridge: University Press.

Van de Pol, J., Volman, M. y Beishuizen, J. (2010). Scaffolding in Teacher-Student Interaction: A Decade of Research. Educational Psychology Review, 22(3), 271-296.

Windschitl, M., Thompson, J. y Braaten, M. (2008). Beyond the Scientific Method: ModelBased Inquiry as a New Paradigm of Preference for School Science Investigations. Science Education, 92(5), 941-967.

Zembal-Saul, C., Blumenfeld, P. y Krajcik, J. (2000). Influence of guided cycles of planning, teaching, and reflection on prospective elementary teachers' science content representations. Journal of Research in Science Teaching, 37(4), 318-339. 


\section{Anexo 1}

Plantilla facilitada a los futuros profesores para resolver situaciones problemáticas abiertas siguiendo la Metodología de Resolución de Problemas como Investigación (MRPI).

\section{MODELO INVESTIGATIVO PARA LA RESOLUCIÓN DE SITUACIONES PROBLEMÁTICAS (MRPI)}

\section{REPRESENTACIÓN DE LA SITUACIÓN PROBLEMÁTICA}

\section{Análisis cualitativo de la situación problemática}

Comprensión y representación de la situación (marco teórico de referencia)

Reformulación del problema en términos operativos

Restricción de condiciones

\section{Emisión de hipótesis}

Emisión de hipótesis acerca de los factores que puedan determinar la magnitud buscada

RESOLUCIÓN DEL PROBLEMA

\section{Diseño de la experimentación y/o estrategia de resolución}

Identificación y control de variables

Determinación de las magnitudes a medir, de los datos, materiales y aparatos requeridos para la solución de la situación problemática

Representación gráfica o esquemática del diseño

Posible establecimiento de analogías con situaciones tratadas anteriormente

Toma de decisiones para la resolución del problema

\section{Desarrollo de la experimentación y/o resolución del problema}

Llevar a cabo el diseño

Descripción del proceso seguido: las observaciones, las medidas, el registro de datos, etc.

\section{ANÁLISIS DEL PROBLEMA}

\section{Análisis de resultados}

Búsqueda de regularidades en los datos

Interpretación de resultados a la luz de las hipótesis y del marco teórico utilizado

Obtención de resultados numéricos, comprobación de unidades, análisis de los órdenes de magnitud, etc. 


\section{Anexo 2}

La siguiente tabla contiene la definición de los niveles de logro para las Dimensiones de la Competencia científica (DC) consideradas en el estudio. Las descripciones son válidas para ambos problemas: P1, ¿Qué puede ocurrir cuando dos sustancias se ponen en contacto? y P3, ¿Qué puede ocurrir cuando se calienta una sustancia? Los niveles se definen entre 0 («no responden o sus aportaciones son irrelevantes») y 4 («respuesta muy satisfactoria»), según se explica en el artículo. El análisis es fruto de la discusión conjunta y el consenso entre los investigadores.

Tabla A. Definición de niveles de logro para las dimensiones competenciales (DC)

\begin{tabular}{|c|c|}
\hline Nivel & Descripción \\
\hline \multicolumn{2}{|r|}{ DC1.1 Representación cualitativa del problema (marco teórico) } \\
\hline 1 & $\begin{array}{l}\text { Describen conceptos básicos como sustancia (elemento/compuesto), mezcla o calor, pero } \\
\text { no centran el marco teórico en explicar la idea del cambio físico o químico. }\end{array}$ \\
\hline 2 & $\begin{array}{l}\text { Además de lo anterior, introducen la idea de cambio físico o químico, aunque de forma } \\
\text { genérica (sin explicar aspectos como la reordenación atómica, la (ir)reversibilidad de los } \\
\text { fenómenos y su relación con que los cambios sean físicos o químicos). }\end{array}$ \\
\hline 3 & $\begin{array}{l}\text { Explican conceptos como sustancia (elemento/compuesto), mezcla, estado de agrega- } \\
\text { ción, cambios físicos y químicos -y posibles indicadores de unos u otros-, calor, etc., pero } \\
\text { no desarrollan las posibilidades de cambio físico o químico en el contexto del problema } \\
\text { (contacto o calentamiento). }\end{array}$ \\
\hline 4 & $\begin{array}{l}\text { Describen adecuadamente todos los conceptos anteriores y, además, aportan un análisis } \\
\text { de posibles cambios físicos o químicos al producirse el contacto entre dos sustancias o su } \\
\text { calentamiento. }\end{array}$ \\
\hline \multicolumn{2}{|r|}{ DC1.2 Reformulación del problema } \\
\hline 1 & No operativizan el problema, tan solo cambian algunos términos de la pregunta inicial. \\
\hline 2 & $\begin{array}{l}\text { Utilizan términos operativos para concretar el enunciado, pero su reformulación no per- } \\
\text { mite completar una investigación sobre las situaciones problemáticas (p.ej., en P3 se ciñe } \\
\text { a un único estado de agregación), o bien resulta algo incoherente con el marco teórico } \\
\text { (p.ej., identificar «puesta en contacto» con «mezcla» en P1). }\end{array}$ \\
\hline 3 & $\begin{array}{l}\text { Utilizan términos operativos para reformular la situación problemática, pero cometen al- } \\
\text { gunos errores en su formulación. }\end{array}$ \\
\hline 4 & $\begin{array}{l}\text { Reformulan adecuadamente el problema, que consistirá en identificar -en base a crite- } \\
\text { rios establecidos- los cambios físicos y químicos que suceden al calentar o poner en con- } \\
\text { tacto las sustancias. }\end{array}$ \\
\hline \multicolumn{2}{|r|}{ DC2 Emisión de hipótesis } \\
\hline 1 & Emiten hipótesis no relacionadas con los objetivos del problema. \\
\hline 2 & $\begin{array}{l}\text { Emiten hipótesis acordes a la situación problemática, aunque resultan incompletas en } \\
\text { relación con los objetivos del problema, o bien reflejan alguna incoherencia con el marco } \\
\text { teórico. }\end{array}$ \\
\hline 3 & $\begin{array}{l}\text { Escriben enunciados coherentes con el problema, pero contienen alguna ambigüedad o } \\
\text { no están expresados en términos de hipótesis falsables (sí/no). }\end{array}$ \\
\hline 4 & Emiten hipótesis coherentes con el problema y correctamente formuladas. \\
\hline \multicolumn{2}{|r|}{ DC3.1 Identificación y control de variables } \\
\hline 1 & $\begin{array}{l}\text { Identifican variables incoherentes con los objetivos del problema, o no identifican expre- } \\
\text { samente variables independientes, dependientes y de control (aunque pueden conside- } \\
\text { rarlas implícitamente en sus diseños experimentales). }\end{array}$ \\
\hline 2 & Identifican correctamente la variable dependiente (VD), en coherencia con sus hipótesis. \\
\hline 3 & $\begin{array}{l}\text { Identifican correctamente las variables dependiente (VD) e independiente (VI), en cohe- } \\
\text { rencia con sus hipótesis. }\end{array}$ \\
\hline 4 & $\begin{array}{l}\text { Explicitan correctamente las variables dependiente (VD) e independiente (VI). Además, } \\
\text { verbalizan algunas variables de control (VC). }\end{array}$ \\
\hline
\end{tabular}


Tabla A. Continuación

\begin{tabular}{|c|c|c|}
\hline ivel & \multicolumn{2}{|c|}{ Descripción } \\
\hline \multicolumn{3}{|c|}{ DC3.2 Toma de decisiones para el problema } \\
\hline 1 & \multicolumn{2}{|c|}{$\begin{array}{l}\text { Describen cómo poner en contacto o calentar las sustancias, pero no especifican los cri- } \\
\text { terios de resolución. }\end{array}$} \\
\hline 2 & \multicolumn{2}{|c|}{$\begin{array}{l}\text { Además de la descripción experimental, explican uno de los siguientes criterios de reso- } \\
\text { lución: i) cambio en las propiedades características como indicador de cambio químico, ii) } \\
\text { (ir)reversibilidad como indicador de cambio físico (o químico), iii) emisión de gases como } \\
\text { indicador de cambio químico / aspectos cinéticos asociados al contacto entre sustancias. }\end{array}$} \\
\hline 3 & \multicolumn{2}{|c|}{$\begin{array}{l}\text { Además de la descripción experimental, explican dos de los criterios anteriores de reso- } \\
\text { lución. }\end{array}$} \\
\hline 4 & \multicolumn{2}{|c|}{$\begin{array}{l}\text { Además de la descripción experimental, explican los tres criterios anterio } \\
\text { ción. }\end{array}$} \\
\hline \multicolumn{3}{|r|}{ ión } \\
\hline 1 & \multicolumn{2}{|c|}{$\begin{array}{l}\text { Resuelven de forma incorrecta el problema, o bien los pasos seguidos resultan claramen } \\
\text { te insuficientes para responder la pregunta (p.ej., en P1 se centran solo en el color, y er } \\
\text { P3 describen únicamente uno de los tres procesos de cambio). }\end{array}$} \\
\hline & \multicolumn{2}{|c|}{$\begin{array}{l}\text { Resuelven adecuadamente el problema, aunque el registro de los datos/observaciones } \\
\text { no es completo en dos de los siguientes aspectos: }\end{array}$} \\
\hline 2 & & Cambio físico (fusión) \\
\hline & P1 Solubilidad de las sustancias intervinientes & P3 Cambio químico (descomposición) \\
\hline & Determinar reactivos limitante y excedente & \\
\hline 3 & \multicolumn{2}{|c|}{$\begin{array}{l}\text { Resuelven adecuadamente el problema, aunque el registro de los datos/observaciones } \\
\text { para uno de los aspectos anteriores no es completo. }\end{array}$} \\
\hline 4 & \multicolumn{2}{|c|}{$\begin{array}{l}\text { Resuelven correctamente el problema, considerando los distintos contenidos implicados } \\
\text { y aportando datos/observaciones experimentales para todos ellos. }\end{array}$} \\
\hline \multicolumn{3}{|c|}{ DC5 Análisis de resultados } \\
\hline 1 & \multicolumn{2}{|c|}{$\begin{array}{l}\text { Plantean un análisis final incorrecto, que no responde a la pregunta de investigación, o } \\
\text { bien omiten referencias a las hipótesis en base a sus datos experimentales. }\end{array}$} \\
\hline 2 & \multicolumn{2}{|c|}{$\begin{array}{l}\text { Describen si sus hipótesis se han verificado o no, e incluyen algo de información al res- } \\
\text { pecto. Sin embargo, no especifican los criterios experimentales que les permiten corro- } \\
\text { borar sus hipótesis -argumentación incompleta-. }\end{array}$} \\
\hline 3 & \multicolumn{2}{|c|}{$\begin{array}{l}\text { Plantean un análisis final con referencia a las hipótesis, a las pruebas experimentales que } \\
\text { permiten corroborarlas y a sus marcos teóricos. }\end{array}$} \\
\hline 4 & \multicolumn{2}{|c|}{$\begin{array}{l}\text { Analizan correctamente sus resultados, haciendo referencia a las hipótesis, a las pruebas } \\
\text { experimentales y a los marcos teóricos. Además, incluyen representaciones microscó- } \\
\text { picas de lo ocurrido utilizando un modelo atómico, y las relacionan con las ecuaciones } \\
\text { químicas correspondientes. }\end{array}$} \\
\hline
\end{tabular}

